\title{
Application of Exopolysaccharide-Producing Cultures in Reduced-Fat Cheddar Cheese: Cryo-Scanning Electron Microscopy Observations*
}

\author{
A. N. Hassan and S. Awadt \\ Minnesota-South Dakota Dairy Foods Research Center, Dairy Science Department, \\ South Dakota State University, Brookings 57007
}

\begin{abstract}
The microstructure of reduced- and full-fat Cheddar cheeses made with exopolysaccharide (EPS)-producing and nonproducing cultures was observed using cryoscanning electron microscopy. Fully hydrated cheese samples were rapidly frozen in liquid nitrogen slush $\left(-207^{\circ} \mathrm{C}\right)$ and observed in their frozen hydrated state without the need for fat extraction. Different EPS-producing cultures were used in making reduced-fat Cheddar cheese. Full-fat cheese was made with a commercial EPS-nonproducing starter culture. The cryo-scanning electron micrographs showed that fat globules in the fully hydrated cheese were surrounded by cavities. Serum channels and pores in the protein network were clearly observed. Young (1-wk-old) full-fat cheese contained wide and long fat serum channels, which were formed because of fat coalescence. Such channels were not observed in the reduced-fat cheese. Young reducedfat cheese made with EPS-nonproducing cultures contained fewer and larger pores than did reduced-fat cheese made with a ropy strain of Lactococcus lactis ssp. cremoris (JFR1), which had higher moisture levels. A 3-dimensional network of EPS was observed in large pores in cheese made with JFR1. Major changes in the size and distribution of pores within the structure of the protein network were observed in all reduced-fat cheeses, except that made with JFR1, as they aged. Changes in porosity were less pronounced in both the full-fat and the reduced-fat cheeses made with JFR1.
\end{abstract}

(Key words: Cheddar, exopolysaccharides, cryo-scanning electron microscopy, microstructure)

Received May 1, 2005.

Accepted August 29, 2005.

Corresponding author: Ashraf N. Hassan; e-mail: Ashraf.Hassan@ sdstate.edu.

*Published with the approval of the director of the South Dakota Agricultural Experiment Station as Publication Number 3474 of the Journal Series. This research was supported in part by MinnesotaSouth Dakota Dairy Foods Research Center, Brookings, South Dakota, and Midwest Dairy Association, St. Paul, Minnesota.

$\dagger$ Current address: Department of Dairy Science and Technology, Faculty of Agriculture, Alexandria University, Egypt.
Abbreviation key: EPS = exopolysaccharide, FFC = full-fat control cheese, RF-JFR1 = reduced-fat cheese made with the ropy culture Lactococcus lactis ssp. cremoris JFR1, SEM = scanning electron microscopy.

\section{INTRODUCTION}

Scanning electron microscopy (SEM) is a useful tool for providing information on microstructure of dairy products, which assists researchers in understanding factors affecting functional, sensory, and physical properties. This technique has been used to study microstructure of different types of cheese such as Cheddar (Metzger and Mistry, 1995), Mozzarella (McMahon et al., 1999), soft cheese (Guerzoni et al., 1999), cream cheese (Sainani et al., 2004), and process cheese (Raval and Mistry, 1999). Cryo-SEM is recommended for studying the microstructure of samples containing high moisture levels (Serp et al., 2002a,b; Hassan et al., 2003). Unlike conventional SEM, the cryo-SEM technique does not require chemical fixation or fat extraction that might induce artifacts. Cheese is a 3-dimensional protein network in which moisture and fat are trapped; therefore, cryo-SEM is valuable when observation of different cheese components is desirable.

Exopolysaccharides (EPS)-producing cultures have been used to modify texture of fermented dairy products (Hassan et al., 1996; Hassan and Frank, 1997; Perry et al., 1997, 1998), but the mechanism by which EPS affects the textural and functional properties of dairy products is not fully understood. Part of the problem was the unavailability of an appropriate microscopy technique. Exopolysaccharides contain about $95 \%$ water, and sample preparation before observation by conventional electron microscopy changes the initial structure and distribution of EPS (Kalab, 1993; Serp et al., 2002a,b). Hassan et al. (2003) used cryo-SEM to visualize the microstructure of soft cheeses and milk fermented with different EPS-producing and nonproducing cultures. Their images showed that EPS and milk fat were visible in pores within the protein network. Exopolysaccharides appeared as a fully hydrated network segregated from the protein network. 
Table 1. Cultures used in this study.

\begin{tabular}{lll}
\hline Strain & Description & Source/Reference \\
\hline Streptococcus thermophilus CHCC 3534 & Moderately ropy & Chr. Hansen, Denmark \\
Streptococcus thermophilus CHCC 5842 & Exopolysaccharide-negative genetic variant of CHCC3534 & Chr. Hansen, Denmark \\
Lactococcus lactic spp. cremoris JFR1 & Highly ropy & Hassan et al., 2003 \\
Streptococcus thermophilus Slab & Capsule-forming nonropy & Hassan et al., 1995a \\
\hline
\end{tabular}

In a companion study, both full-fat cheese and reduced-fat cheese made with a ropy strain of Lactococcus lactis spp. cremoris JFR1 had similar textural and melting characteristics (Awad et al., 2005b). In addition, changes in their physical properties during ripening followed the same pattern (Awad et al., 2005b). The physical and functional properties of cheese are governed by its microstructure (Kalab et al., 1987; Pastorino et al., 2003). Therefore, cheese microstructure might explain why this particular strain of Lactococcus lactis spp. cremoris (JFR1) produced reduced-fat cheese with characteristics similar to those of its full-fat counterpart. The objectives of this work were to 1) study microstructure of Cheddar cheese in its fully hydrated state using cryo-SEM, 2) observe microstructural changes in full- and reduced-fat Cheddar cheeses manufacturing with EPS-producing cultures and, 3) relate microscopic observations to the previously reported textural, functional, and viscoelastic properties of reducedand full-fat Cheddar cheeses made with different cultures (Awad et al., 2005b; Hassan et al., 2005).

\section{MATERIALS AND METHODS}

\section{Cultures}

Table 1 shows cultures used in this study. Bacterial strains were maintained at $-80^{\circ} \mathrm{C}$ in $11 \%$ sterile reconstituted skim milk supplemented with $20 \%$ (vol/vol) glycerol. Lactococci and streptococci were grown in M17 broth (Becton Dickinson Co., Sparks, MD), supplemented with $0.5 \%$ (wt/vol) lactose and incubated overnight at $32^{\circ} \mathrm{C}$ (for lactococci) or $39^{\circ} \mathrm{C}$ (for streptococci). Each strain was subcultured ( $1 \% \mathrm{vol} / \mathrm{vol}) 3$ times and then transferred to $11 \%$ reconstituted skim milk for overnight incubation to produce the cheese starter culture. Commercial direct to vat set Cheddar culture (DVS 850) was obtained from Chr. Hansen Lab (Milwaukee, WI).

\section{Cheese Making}

Raw milk was obtained from the Dairy Research and Training Facility at South Dakota State University. Cheddar cheese was manufactured from standardized (2\% reduced fat or $3.6 \%$ full fat) pasteurized $\left(63^{\circ} \mathrm{C}\right.$ for
30 min and cooled to $31^{\circ} \mathrm{C}$ ) milk. The following 6 cheese treatments were made: 1) $\mathbf{F F C}=$ full-fat cheese made using the commercial Cheddar starter culture (DVS $850 ; 0.015 \% \mathrm{wt} / \mathrm{wt}) ; 2$ ) reduced-fat cheese made using the commercial Cheddar starter culture (DVS 850; 0.015\% wt/wt); 3) RF-JFR1 = reduced-fat cheese made with the ropy strain Lactococcus lactis spp. cremoris JFR1 (2\% vol/wt); 4) reduced-fat cheese made with a capsule-forming nonropy Streptococcus thermophilus $(0.4 \% \mathrm{vol} / \mathrm{wt})$ plus the commercial culture $(0.011 \% \mathrm{wt} /$ wt); 5) reduced-fat cheese made with EPS-producing Streptococcus thermophilus CHCC $3534(0.4 \% \mathrm{vol} / \mathrm{wt})$ plus the commercial culture $(0.011 \% \mathrm{wt} / \mathrm{wt})$; and 6$)$ reduced-fat cheese made with the EPS-negative genetic variant of CHCC 3534 (Streptococcus thermophilus CHCC 5842; 0.4\% vol/wt) plus the commercial culture $(0.011 \% \mathrm{wt} / \mathrm{wt})$. The inoculum size was selected, based on the preliminary experimental data, to give the same acidification rate and cheese-making time in all treatments. Cheddar cheese was made as described by Awad et al. (2005a).

\section{Cryo-SEM}

Two cheese samples from 1 replication were examined in this study. Several microscopic fields were observed and representative images have been selected. Samples $(1 \mathrm{~cm} \times 1 \mathrm{~cm})$ were mounted onto holders and pumped into liquid nitrogen slush at $-207^{\circ} \mathrm{C}$. Frozen specimens were transferred under vacuum into an attached preparation chamber where they were fractured using a gold scalpel blade. Fractured surfaces were etched at $-80^{\circ} \mathrm{C}$ for $15 \mathrm{~min}$ and coated with 300 $\AA$ of sputtered gold. The specimens were transferred under vacuum onto the cold stage where they were maintained at $-95^{\circ} \mathrm{C}$, and imaged using SEM (Leo Electron Microscopy Inc., Thornwood, NY) at $4 \mathrm{kV}$.

\section{RESULTS AND DISCUSSION}

Figure 1 shows the microstructure of young (1 wk old) full- and reduced-fat Cheddar cheeses made with EPS-producing and nonproducing cultures. Fat globules in all cheeses were surrounded by cavities. Such zones do not seem to be formed as a result of differences in thermal expansion/contraction between fat and wa- 
A

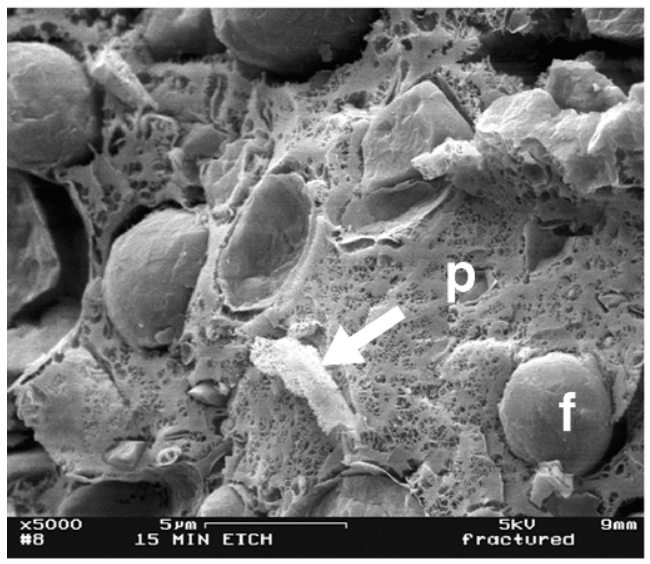

C

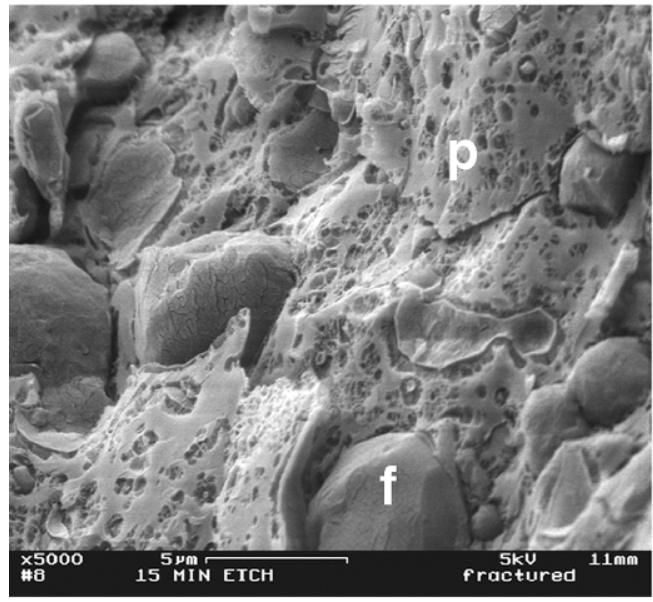

E

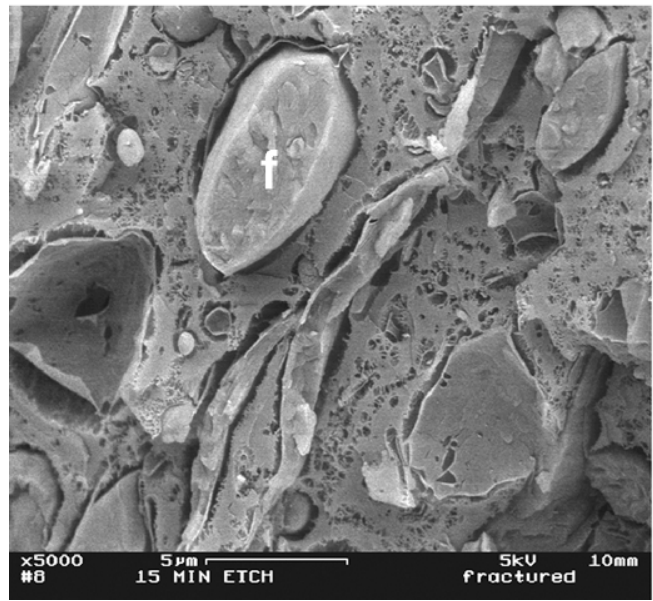

B

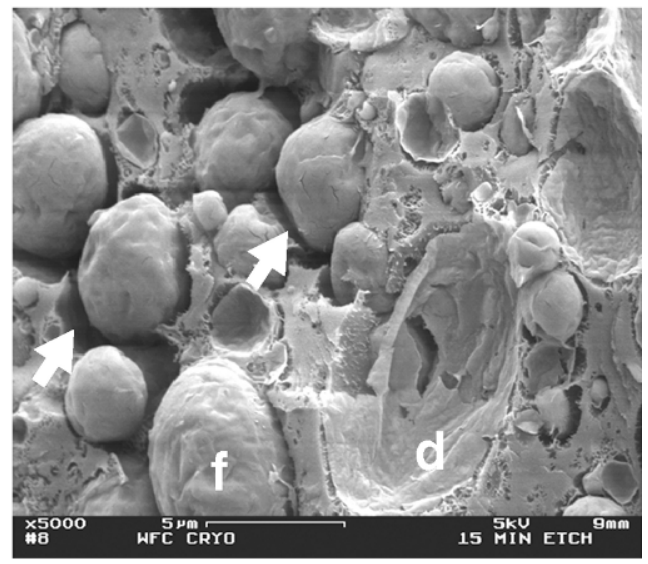

D

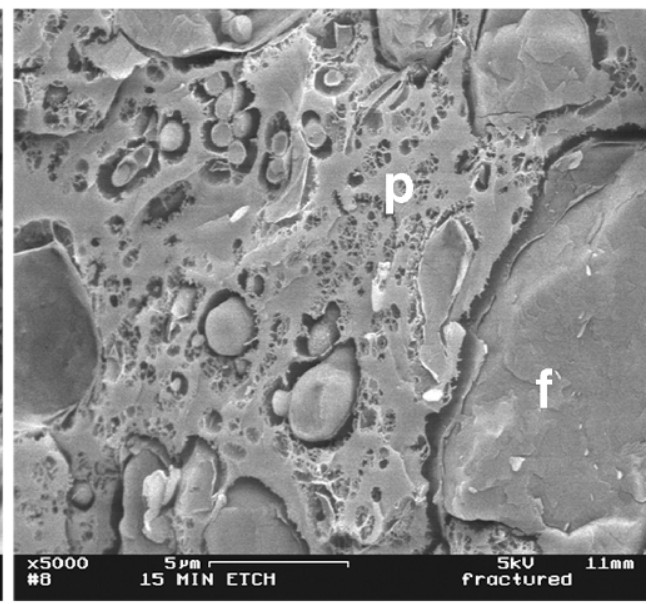

$\mathbf{F}$

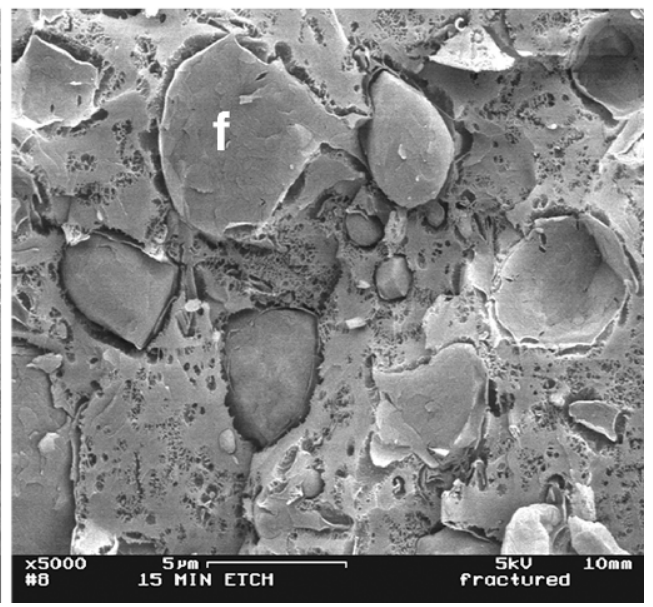

Figure 1. Microstructure of reduced-fat (A, C, D, E, F) and full-fat (B) cheeses made with different exopolysaccharide (EPS)-producing (A, E, F) and nonproducing (C, D) cultures. A = RF-JFR1 (reduced-fat cheese made with the highly ropy culture Lactococcus lactis ssp. cremoris JFR1, B = FFC (full-fat control), C = RFC (reduced-fat control), D = RF-5842 (reduced-fat cheese made with the EPS-negative genetic variant Streptococcus thermophilus CHCC 5842), E = RF-Slab (reduced-fat cheese made with the capsule-forming nonropy strain Streptococcus thermophilus Slab, F = RF-3534 (reduced-fat cheese made with the moderately ropy strain Streptococcus thermophilus CHCC 3534). Arrow in panel A indicates EPS and in B indicates fat-serum channels; $p=$ background protein network; $f=f a t ; d=d e p r e s s i o n$ originally occupied by a fat globule and formed because of surface fracture. 
Table 2. Chemical composition (\%) and $\mathrm{pH}$ of full-fat and reduced-fat Cheddar cheese made with different exopolysaccharide-producing and nonproducing cultures. ${ }^{1}$

\begin{tabular}{|c|c|c|c|c|c|c|}
\hline \multirow[b]{2}{*}{ Treatment $^{2}$} & \multicolumn{6}{|c|}{ Composition and characteristics } \\
\hline & $\mathrm{pH}$ & Protein & Fat & Moisture & Salt-in-moisture & $\mathrm{MNFS}^{3}$ \\
\hline $\mathrm{FFC}$ & 5.24 & $25.14^{\mathrm{c}}$ & $32.07^{\mathrm{a}}$ & $39.75^{\mathrm{d}}$ & $3.70^{\mathrm{a}}$ & $58.51^{\mathrm{a}}$ \\
\hline RFC & 5.24 & $32.23^{\mathrm{a}}$ & $20.20^{\mathrm{b}}$ & $42.64^{\mathrm{c}}$ & $3.35^{\mathrm{b}}$ & $53.44^{\mathrm{c}}$ \\
\hline RF-JFR1 & 5.20 & $28.47^{\mathrm{b}}$ & $18.90^{\mathrm{c}}$ & $47.33^{\mathrm{a}}$ & $3.06^{\mathrm{c}}$ & $58.36^{\mathrm{a}}$ \\
\hline RF-Slab & 5.20 & $30.54^{\mathrm{a}}$ & $19.88^{\mathrm{b}}$ & $43.98^{\mathrm{b}}$ & $3.27^{\mathrm{b}}$ & $54.90^{\mathrm{b}}$ \\
\hline RF-3534 & 5.22 & $30.90^{\mathrm{a}}$ & $20.08^{\mathrm{b}}$ & $43.43^{\mathrm{c}}$ & $3.36^{\mathrm{b}}$ & $54.35^{\mathrm{bc}}$ \\
\hline RF-5842 & 5.25 & $32.28^{\mathrm{a}}$ & $20.04^{\mathrm{b}}$ & $42.74^{\mathrm{c}}$ & $3.35^{\mathrm{b}}$ & $53.44^{\mathrm{c}}$ \\
\hline \multicolumn{7}{|c|}{ 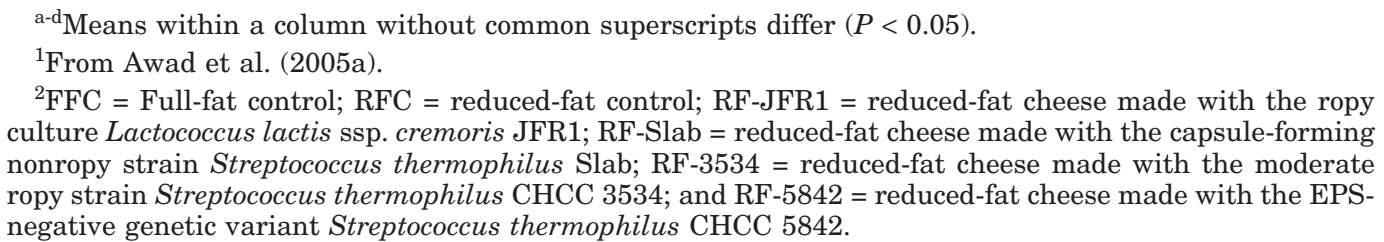 } \\
\hline
\end{tabular}

ter during freezing and etching because their presence was confirmed in unfrozen cheese specimens by fluorescence microscopy (data not shown). In addition, the freezing technique used in this study (freezing under vacuum in liquid nitrogen slush at $-207^{\circ} \mathrm{C}$ ) should stabilize the initial structure. The presence of spaces around fat globules in young Cheddar cheese indicated a lack of interaction between fat and protein network due to the presence of expressible serum. In conventional SEM, fat is extracted before microscopic observations. Therefore, both fat and pores containing serum appear as cavities (Anderson and Mistry, 1994; Metzger and Mistry, 1995). One of the advantages of the cryoSEM technique used in this study is the ability to directly observe fat globules within the protein matrix. Pores occupied by serum could be easily identified in the micrographs (Figure 1). Different sized fat globules embedded in the continuous protein network were observed in the full-fat cheese (Figure 1B). Channels containing fat and serum (expressible water) were also present in this type of cheese. Such fat-serum channels were formed because of fat coalescence, which was limited in the reduced-fat types. Guinee et al. (2000) also observed that fat reduction resulted in a decrease in the extent of fat globule clumping and coalescence. Because of the volume exclusion effect of the fat globules, protein in the full-fat cheese occupied less space (Figure 1B). The relatively high level of protein in the reducedfat cheese compared to that in the full-fat type (Table 2 ) produced wider areas of protein network unoccupied with fat (Figure 1).

Two types of porosity could be seen in the micrographs presented in Figure 1. In full-fat cheese, the porosity was mainly produced by serum channels between protein strands. However, pores within the background protein network were more obvious in the re- duced-fat types. The higher moisture level in reducedfat cheese compared to that in the full-fat (Table 2) cheese might be responsible for the more porous network structure observed in this type of cheese. Size and distribution of the pores within the protein network differed among reduced-fat cheeses and were affected by the type of culture used. Young reduced-fat cheese made with EPS-negative cultures contained fewer and larger pores than did cheese made with the ropy culture JFR1, which had higher moisture levels (Table 2). In addition, protein strands were wider in the reduced-fat cheese made with no EPS than those in the EPS-positive cheeses. Such strands may have resisted compression more and produced the firm structure of the young reduced-fat cheese made with no EPS (Awad et al., 2005b). The electron microscopy micrographs correlated well with the texture analysis data (Awad et al., 2005b). Disturbance of protein-protein interactions by the fat-serum channels in full-fat cheese and the high porosity of the background protein network (resulting from the high moisture level) in the EPS-positive cheese (RF-JFR1) seemed to be responsible for the reduced firmness in these types of cheese.

Microstructure of Cheddar cheese aged for $6 \mathrm{mo}$ is shown in Figure 2. High magnification was used to observe details of the structure of protein network in the mature cheese because we observed a significant reduction in the size of pores in most cheeses. The overall structure of aged reduced-fat cheese made with the ropy culture JFR1 was very similar to that of the fullfat cheese. Both cheeses contained pores that were larger than those in all other reduced-fat cheeses. Whereas large pores contained fat globules in FFC, they were associated with the presence of either fat or EPS in cheese made with the EPS-positive culture JFR1. The difference in microstructure between RF-JFR1 and 
A

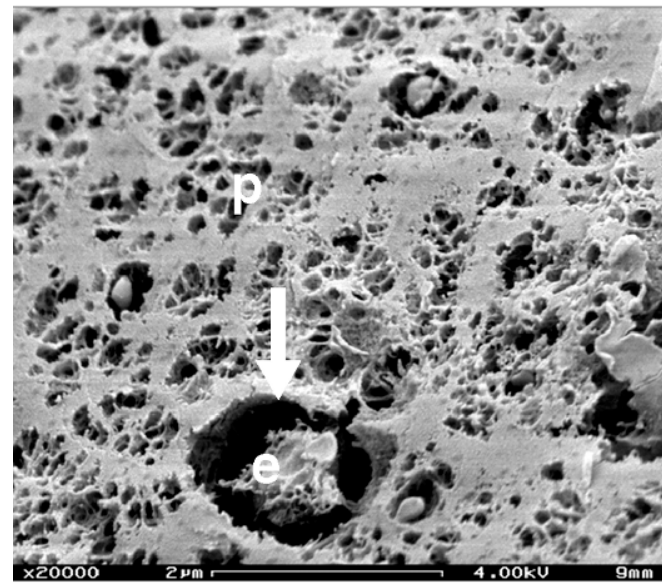

C

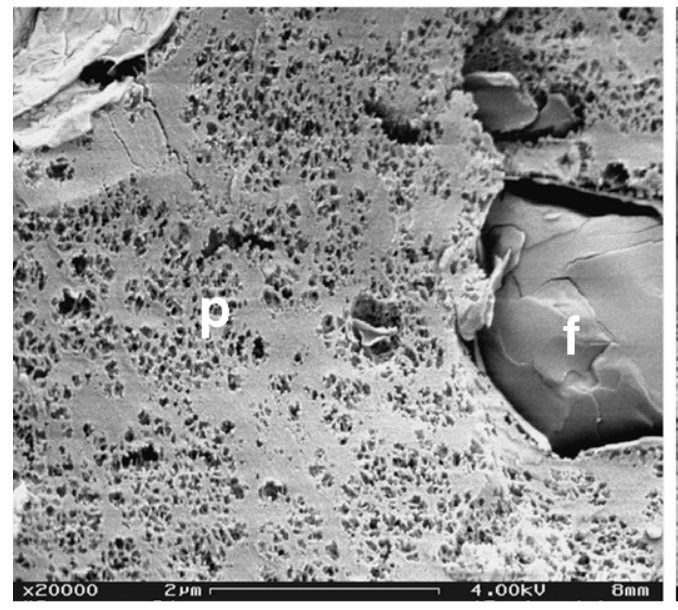

E

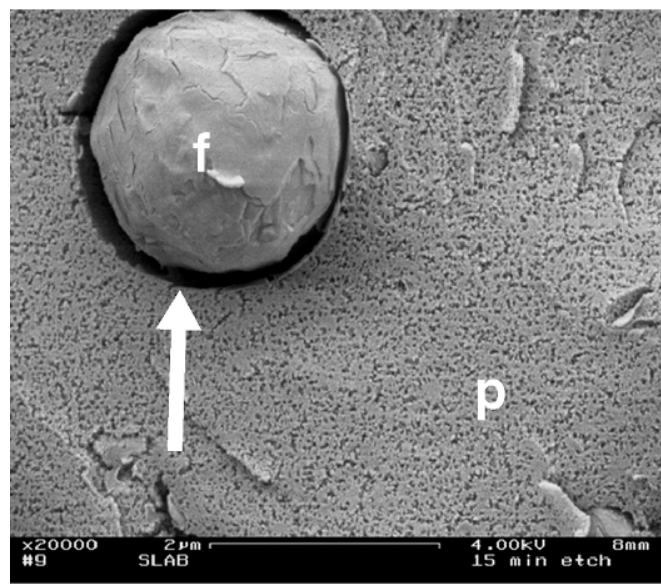

B

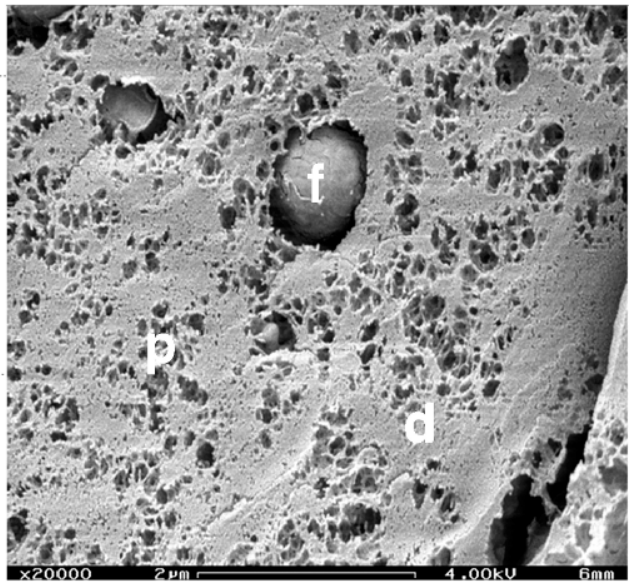

D

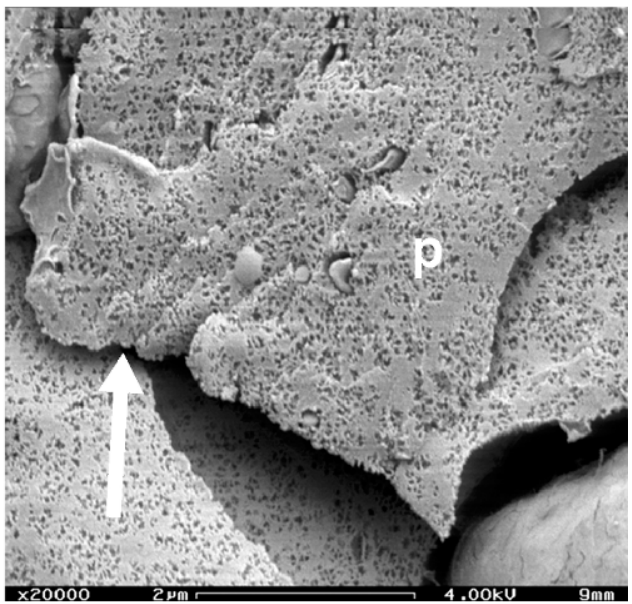

F

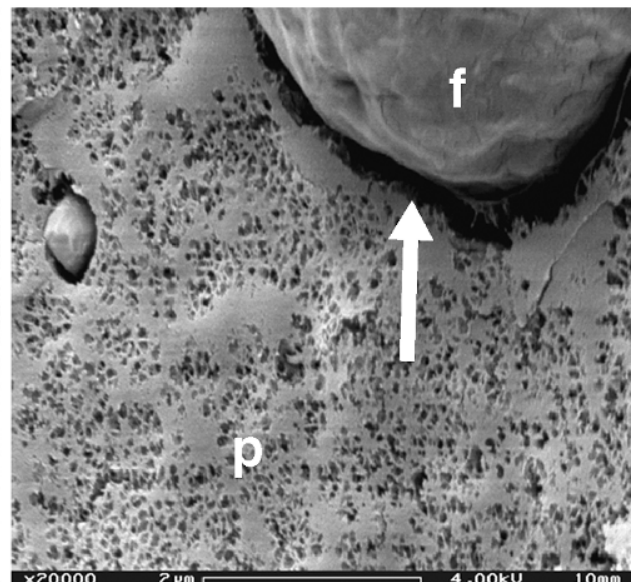

Figure 2. Microstructure of 6-mo-old reduced-fat (A, C, D, E, F) and full-fat (B) cheeses made with different exopolysaccharide (EPS)producing (A, E, F) and nonproducing (C, D) cultures. A = RF-JFR1 (reduced-fat cheese made with the highly ropy culture Lactococcus lactis ssp. cremoris JFR1, B = FFC (full-fat control), C = RFC (reduced-fat control), D = RF-5842 (reduced-fat cheese made with the EPSnegative genetic variant Streptococcus thermophilus CHCC 5842), E = RF-Slab (reduced-fat cheese made with the capsule-forming nonropy strain Streptococcus thermophilus Slab, F = RF-3534 (reduced-fat cheese made with the moderately ropy strain Streptococcus thermophilus CHCC 3534). Arrow in panel A indicates a large pore occupied by EPS and in D indicates a fracture in the protein network; arrows in panels $\mathrm{E}$ and $\mathrm{F}$ indicate cavities surrounding fat globules; $\mathrm{p}=$ background protein network; $\mathrm{f}=\mathrm{fat}$. 
FFC is that pores were more interconnected in the former cheese, which produced a more open appearance. The presence of EPS could be responsible for this observation. Exopolysaccharide-producing cultures have been used in making low-fat, acid-coagulated soft cheese, the porosity of which was associated with the presence of EPS (Hassan et al., 2004). In acid-coagulated cheese, enough time is given to the culture to produce EPS before the gelation initiates. This EPS interferes with the network formation, which is reflected in the physical properties of the final cheese. However, in Cheddar cheese, most of the EPS is produced after the protein network has already been formed (because of the relatively short milk ripening period). Exopolysaccharides produced before milk gelation would act as nuclei for the formation of large pores (Hassan et al., 1995b). If EPS were produced after the network has already been formed, it would still be associated with the serum phase where the producers (bacterial cells) are located. This EPS might reduce proteinprotein interactions, which could lead to reduced rigidity (Awad et al., 2005b). Only EPS associated with large pores was visible in Figure 2. Exopolysaccharides produced by 2 of the cultures used in this study (S3534 and JFR1) formed a 3-dimensional network (Hassan et al., 2003). A network of EPS is also seen in Cheddar cheese (Figure 2A).

Fat-free areas (background protein network) within the protein network in reduced-fat cheese (Figure 2, D and E), formed layers that were not completely connected due to the absence of the lubricating effect of fat. Such layered structure was less pronounced in the protein network of cheese made with either full fat or the ropy culture (JFR1; Figure 2, A and B) due to the presence of high levels of fat or EPS, both of which acted as lubricants.

Smaller cavities surrounding fat globules and more direct contact between fat and protein network were observed in the 6-mo-old full fat cheese compared with the young cheese, which indicated a reduction in the amount of the expressible water as cheese aged. This observation is consistent with findings of other researchers (Guo and Kindstedt, 1995; Ramkumar et al., 1997; McMahon et al., 1999). The reduction in the expressible serum that resulted in more fat-protein interactions might have been responsible for the increase in firmness of FFC as it aged (Awad et al., 2005b). No large differences were observed in the microstructure between mature and young cheese made with the ropy culture JFR1. However, aged reduced-fat cheeses made with no EPS contained smaller pores compared with their young counterparts. Changes in the pore size indicated that redistribution of the moisture in those cheeses was occurring during ripening. The distribution of the moisture and other components in cheese during ripening can be explained by system thermodynamics. During ripening, the cheese system reaches equilibrium conditions that attain a minimum energy (Ramkumar et al., 1997). In comparing Figures 1 and 2, we concluded that reduced-fat cheeses, except RF-JFR1, underwent more extensive water redistribution than both FFC and RF-JRF1. The large changes in the distribution of the moisture phase (and porosity) in reducedfat cheeses, except RF-JFR1, during ripening led to significant changes in their textural and functional properties (Awad et al., 2005b). Although a significant reduction in the firmness of reduced-fat cheeses, except RF-JFR1, took place during aging, a slight increase in firmness of FFC and RF-JFR1 was observed. Cheeses underwent major microstructural changes during aging (all reduced-fat cheeses except RF-JFR1) were those that showed the most significant changes in their physical properties (Awad et al., 2005b).

\section{CONCLUSIONS}

Cryo-SEM is a useful tool for studying microstructure of Cheddar cheese. This technique allowed observations of changes in porosity and distribution of fat globules and EPS within the cheese matrix. Because of the fully hydrated state of cheese specimens observed with cryoSEM, this technique provides a more accurate picture of microstructure of cheese and helps researchers understand factors affecting the physical and functional properties of cheese. Major differences in the porosity of the protein network between young and aged reduced-fat Cheddar cheeses were observed. This indicated that extensive water redistribution took place in these cheeses during ripening. However, microstructural changes during ripening of both full-fat cheese and reduced-fat cheese made with a ropy strain of Lactococcus lactis ssp. cremoris were less pronounced. The degree of water redistribution during ripening seemed to be related to the level of free water in young cheese.

\section{ACKNOWLEDGMENTS}

This work was supported in part by the MinnesotaSouth Dakota Dairy Foods Research Center. The authors would like to thank John Shields, Assistant Director of the Center for Ultrastructural Research at The University of Georgia (Athens, GA) for his assistance with cryo-scanning electron microscopy.

\section{REFERENCES}

Anderson, D. L., and V. V. Mistry. 1994. Reduced fat Cheddar cheese from condensed milk. 2. Microstructure. J. Dairy Sci. 77:7-15. 
Awad, S., A. N. Hassan, and F. Halaweish. 2005a. Application of exopolysaccharide-producing cultures in reduced fat Cheddar cheese: Composition and proteolysis. J. Dairy Sci. 88:4195-4203.

Awad, S., A. N. Hassan, and K. Muthukumarappan. 2005b. Application of exopolysaccharides-producing cultures in reduced fat Cheddar cheese: Texture and melting properties. J. Dairy Sci. 88:4204-4213.

Guerzoni, M. E., L. Vannini, C. Chaves Lopez, R. Lanciotti, G. Suzzi, and A. Gianotti. 1999. Effect of high pressure homogenization on microbial and chemico-physical characteristics of goat cheeses. J. Dairy Sci. 82: 851-862.

Guinee, T. P., M. A. E. Auty, and M. A. Fenelon. 2000. The effect of fat content on the rheology, microstructure and heat induced functional characteristics of Cheddar cheese. Int. Dairy J. 10:277-288.

Guo, M., and P. Kindstedt. 1995. Age-related changes in the water phase of Mozzarella cheese. J. Dairy Sci. 78:2099-2107.

Hassan, A. N., S. Awad, and K. Muthukumarappan. 2005. Effects of exopolysaccharide-producing cultures on the viscoelastic properties of reduced fat Cheddar cheese. J. Dairy Sci. 88:4221-4227.

Hassan, A. N., M. Corredig, J. R. Frank, and M. Elsoda. 2004. Microstructure and rheology of an acid coagulated cheese (Karish) made with an exopolysaccharide-producing Streptococcus thermophilus strain and its exopolysaccharide non-producing genetic variant. J. Dairy Res. 71:116-120.

Hassan, A. N., and J. F. Frank. 1997. Modification of microstructure and texture of rennet curd by using a capsule-forming non-ropy lactic cultures. J. Dairy Res. 64:115-121.

Hassan, A. N., J. F. Frank, and M. El-Soda. 2003. Observation of bacterial exopolysaccharides in dairy products using cryo-scanning electron microscopy. Int. Dairy J. 13:755-762.

Hassan, A. N., J. F. Frank, M. A. Farmer, K. A. Schmidt, and S. I. Shalabi. 1995a. Observation of encapsulated lactic acid bacteria using confocal scanning laser microscopy. J. Dairy Sci. 78:2624-2628.

Hassan, A. N., J. F. Frank, M. A. Farmer, K. A. Schmidt, and S. I. Shalabi. 1995b. Formation of yogurt microstructure and threedimensional visualization as determined by confocal scanning laser microscopy. J. Dairy Sci. 78:2629-2636.
Hassan, A. N., J. F. Frank, K. A. Schmidt, and S. I. Shalabi. 1996. Textural properties of yogurt made with encapsulated nonropy lactic cultures. J. Dairy Sci. 79:2098-2103.

Kalab, M. 1993. Practical aspects of electron microscopy in dairy research. Food Struct. 12:95-114.

Kalab M., J. Yun, and S. H. Yiu. 1987. Textural properties and microstructure of process cheese food rework. Food Microstruct. 6:181-192.

McMahon, D., R. L. Fife, and C. Oberg. 1999. Water partitioning in Mozzarella cheese and its relationship to cheese meltability. J. Dairy Sci. 82:1361-1369.

Metzger, L. E., and V. V. Mistry. 1995. A new approach using homogenization of cream in the manufacture of reduced fat Cheddar cheese. 2. Microstructure, fat globule distribution, and free oil. J. Dairy Sci. 78:1883-1895.

Pastorino, A. J., C. L. Hansen, and D. J. McMahon. 2003. Effect of $\mathrm{pH}$ on the chemical composition, and structure-function relationships of Cheddar cheese. J. Dairy Sci. 86: 2751-2760.

Perry, D. B., D. J. McMahon, and C. J. Oberg. 1997. Effect of exopolysaccharide-producing cultures on moisture retention in low-fat Mozzarella cheese. J. Dairy Sci. 80:799-805.

Perry, D. B., D. J. McMahon, and C. J. Oberg. 1998. Manufacture of low-fat Mozzarella cheese using exopolysaccharide-producing starter culture. J. Dairy Sci. 81:563-566.

Ramkumar, C., L. Creamer, K. Johnston, and R. Bennett. 1997. Effect of $\mathrm{pH}$ and time on the quantity of readily available water within fresh cheese curd. J. Dairy Res. 64:123-134.

Raval, D. M., and V. V. Mistry. 1999. Application of ultrafiltered sweet buttermilk in the manufacture of reduced fat process cheese. J. Dairy Sci. 82: 2334-2343.

Sainani, M. R., H. K. Vyas, and P. S. Tong. 2004. Characterization of particles in cream cheese. J. Dairy Sci. 87:2854-2863.

Serp, D., M. Mueller, U. von Stockar, and I. W. Marison. 2002a. Lowtemperature electron microscopy for the study of polysaccharide ultrastructures in hydrogels. I. Theoretical and technical considerations. Biotechnol. Bioeng. 79:243-252.

Serp, D., M. Mueller, U. von Stockar, and I. W. Marison. 2002b. Lowtemperature electron microscopy for the study of polysaccharide ultrastructures in hydrogels. II. Effect of temperature on the structure of $\mathrm{Ca}^{+2}$-alginate beads. Biotechnol. Bioeng. 79:253-259. 